\title{
HIGH ACCURACY ALGORITHMS OF NUMERICAL PREDICTION OF THE MOTION OF SOLAR SYSTEM BODIES
}

\author{
T.V. BORDOVITSYNA AND V.A. SHEFER \\ Research Institute of Applied Mathematics and Mechanics \\ Tomsk University \\ 634050 Tomsk \\ USSR
}

\begin{abstract}
A brief summary of results obtained by the authors for investigations of the efficiency of numerical prediction algorithms for natural and artificial minor bodies using regularizing and stabilizing transformations is given.
\end{abstract}

The problem of high accuracy interpretation of observations of solar system bodies is closely connected with the problem of the accurate prediction of the motion of these objects.

This paper presents a brief summary of results obtained by the authors (Bordovitsyna 1984; Shefer 1989; Bordovitsyna et al. 1989) for the development and investigation of effective algorithms for the numerical prediction of the motion of minor bodies of the solar system. Research on the efficiency of the set of regularizing and stabilizing methods in the problem of numerical prediction of the motion of celestial bodies was carried out by the authors. It was shown that the introduction of integrals of motion, KS-transformation and time transformation $d t=r^{\mathrm{n}} d s$ with $n=1$ in the equations of motion is the most effective way of stabilization and regularization of these equations. These transformations do not destroy the numerical stability of the calculation algorithm and at the same time they stabilize and regularize the equations of motion.

The research on numerical methods has shown that Everhart's implicit single sequence methods and the modified algorithm of rational extrapolation are the most effective ones.

As a result of these investigations, high accuracy algorithms and software were developed. There are two application packages for prediction of the motion of natural minor bodies of the solar system and of artificial satellites.

The research on the efficiency of the application package "The dynamics of minor bodies of the solar system" was carried out on the examples of the motion of the unusual minor planets Icarus and Geographos as well as comets Halley, Honda-Mrkos-Pajdusakova and Gehrels 3.

An analogous research on the application package "The numerical model of artificial satellite motion" was made in solving the problem of numerical prediction of the motion of artificial satellites 
with different kinds of orbital parameters as well as representing observations obtained by the MERTT program.

The accuracy of representation of observations of minor MERT program objects equals $\pm 50 \mathrm{~cm}$.

\section{References}

Bordovitsyna, T.V.: 1984, Modern Numerical Methods in Celestial Mechanics, Nauka , Moscow (in Russian).

Bordovitsyna, T.V., Bykova, L.E., Boronenko, T.S., Tamarov, V.A., Sharkovsky, N.A., and Shmidt, Yu.B.: 1989, Numerical and Semi-analytical Algorithms for Prediction of the Motion of Artificial Satellites, Tomsk State University, Tomsk (in Russian; in press).

Shefer, V.A.: 1989, Celest. Mech. (in press). 